\title{
Comment on "Observation of a Push Force on the End Face of a Nanometer Silica Filament Exerted by Outgoing Light"
}

\author{
Iver Brevik* \\ Department of Energy and Process Engineering, Norwegian University of Science and Technology, N-7491 Trondheim, Norway
}

The Abraham-Minkowski electromagnetic energymomentum tensor problem has been on the agenda since about 1910. The recent experiment of She et al. [1] is in this connection of interest, as it shows how the radiation force from a low-intensity laser yields an inward push force on the end face of a vertical fiber.

But does this experiment measure electromagnetic momentum? In our opinion the answer is no. What is detected is merely the electromagnetic AbrahamMinkowski force density $\mathbf{f}^{A M}=-\left(\varepsilon_{0} / 2\right) E^{2} \nabla n^{2}$ in the surface layer of the filament (or in other regions where $n$ varies). This is not related to the electromagnetic momentum in itself. The electromagnetic force density is $\mathbf{f}=\mathbf{f}^{A M}+\left[\left(n^{2}-1\right) / c^{2}\right] \partial / \partial t(\mathbf{E} \times \mathbf{H})$, and electromagnetic momentum does not appear until the second term in this expression. This is the Abraham term. It is in principle measurable although it is usually small; moreover it simply fluctuates out when averaged over an optical period in a stationary beam.

For illustration, let us assume that a short laser pulse with energy $\mathcal{H}$ falls from vacuum towards the entrance surface of a free-standing fiber ( we ignore gravity). If there is an antireflection film of refractive index $\sqrt{n}$ on the surface, $\mathcal{H}$ is the energy of the pulse in the medium also. The impulse imparted to the surface because of the surface force $f^{A M}$ is $G_{\text {surf }}=\mathcal{H}(n-1) / c$, directed against the beam if $n>1$. When the pulse leaves at the exit surface, a corresponding reverse impulse is imparted. In the Abraham case, one has to take into account the mechanical momentum $G_{\text {mech }}^{A}$ caused by the Abraham term also. One finds $G_{\text {mech }}^{A}=\mathcal{H}\left(n^{2}-1\right) / n c$. The resulting longitudinal displacement of the fiber because of the sum $G_{\text {surf }}+G_{\text {mech }}^{A}$ becomes $\Delta x^{A}=\left(\mathcal{H} / c^{2} \mu\right)(n-1)$, where $\mu=M / L$ is the mass per unit length. As discussed on p. 189 in Ref. [2], $\Delta x^{A} \sim 1$ pm or less, and is clearly non-observable.

In the present case, the fiber is fixed at the upper end. There will be a downward directed impulse imparted to the fiber at the lower end when the pulse leaves. It is very small: taking the flux to be $10 \mathrm{~mW}$ and the pulse duration to be $270 \mathrm{~ms}$, we get $\mathcal{H}=2.7 \mathrm{~mJ}$ resulting in $G_{\text {surf }}=4.5 \mathrm{pN} \cdot \mathrm{s}$ if $n=1.5$. Because of elasticity, there *Electronic address: iver.h.brevik@ntnu.no will be an upward directed recoil in the fiber. (Sideways motion may result from non-axisymmetric elastic conditions.)

We propose finally a modification of the experiment that might be capable of detecting the Abraham force after all (cf. also p. 191 of [2]): Let a long fiber of length $L$ be wound up on a drum of radius $R$ and of small weight. Suspend the drum as a vertical torsional pendulum such that it can oscillate about the $z$ axis with an eigenfrequency $\omega_{0}$. Then send an intensity modulated optical wave through the fiber, such that its harmonic component has the same frequency $\omega_{0}$. We take the incident energy flux in vacuum to be $P^{(i)}=P_{0} \cos ^{2}\left(\frac{1}{2} k_{0} x-\frac{1}{2} \omega_{0} t\right)$, where $k_{0}=\omega_{0} / c, x$ is the longitudinal coordinate, and $P_{0}$ is the unmodulated energy flux averaged over an optical period. With antireflection films on the end surfaces we obtain in the Abraham case a longitudinal force $F^{A}=$ $P_{0}[(n-1) / c n] \sin \left(\frac{1}{2} n k_{0} L\right) \sin \left(\frac{1}{2} n k_{0} L-\omega_{0} t\right)$, whereas in the Minkowski case $F^{M}=-n F^{A}$. These forces give rise to measurable axial torques $N_{z}$ on the drum. Assuming the sheet of fiber on the drum to be thin we obtain, when setting $\sin \left(\frac{1}{2} n k_{0} L\right) \approx \frac{1}{2} n k_{0} L$, in the Abraham case $N_{z}^{A}=\left[(n-1) / 2 c^{2}\right] R L P_{0} \omega_{0} \sin \left(\frac{1}{2} n k_{0} L-\omega_{0} t\right)$. In the Minkowski case, $N_{z}^{M}=-n N_{z}^{A}$. The two predictions are thus quite different.

For definiteness,assume that a YAG laser at $1.06 \mu \mathrm{m}$ produces the incident beam. Assume that a high power of $P_{0}=1 \mathrm{~kW}$ can be transmitted through the fiber, and neglect any losses. Then, with $L=100 \mathrm{~m}, n=1.5, R=$ $10 \mathrm{~cm}, \omega_{0}=10 \mathrm{~s}^{-1}$, we obtain for the predicted torque amplitudes $N_{z}^{A}=2.8 \times 10^{-13} \mathrm{Nm}, N_{z}^{M}=4.2 \times 10^{-13} \mathrm{Nm}$.

The above amplitudes are less than those of Ref. 3] $\left(10^{-12} \mathrm{Nm}\right)$, but of the same order of magnitude as in Ref. [4]. Actually, they are greater than those in the classic experiment of Ref. [5] $\left(10^{-16} \mathrm{Nm}\right)$. Realization of our proposed experiment appears difficult but not impossible.

Finally, it should be mentioned that our Einstein-box argument above implicitly assumed wide lateral dimensions for the pulse. Cf. also the Comment of Mansuripur on this point [6].
[1] W. She, J. Yu, and R. Feng, Phys. Rev. Lett. 101, 243601 (2008).

[2] I. Brevik, Physics Reports 52, 133 (1979).

[3] G. Roosen and C. Imbert, Can. J. Phys. 52, 1903 (1974).

[4] R. V. Jones and J. C. S. Richards, Proc. Roy. Soc. A 221,
480 (1954).

[5] R. A. Beth, Phys. Rev. 50, 115 (1936).

[6] M. Mansuripur, Phys. Rev. Lett. 103, 019301 (2009). 